\title{
BEYOND THE STANDARD MODEL PHYSICS REACH OF THE ATLAS EXPERIMENT
}

\author{
G.Unel ${ }^{a}$ \\ Physics Division, CERN, Geneva 23, Switzerland; and \\ Department of Physics, University of California at Irvine, Irvine, USA. \\ Abstract. A brief summary of the current unsolved probems of the Standard Model \\ of particle physics is presented. The installation and commissioning status of the \\ ATLAS experiment on the LHC accelerator is given. A phenomenological approach \\ is followed to show the physics reach of the ATLAS experiment. Theories beyond \\ the Standard Model are classified according to the fundamental structure they \\ modify: Matter fields, gauge group structure, symmetry breaking mechanism and \\ space-time.
}

\section{The Standard Model and the ATLAS experiment}

The Standard Model (SM) of particle physics is based on four pillars. The first two are the fermion fields which define the matter and the gauge group structure which describes the fermion interactions. The interactions are carried out by mediator particles, the gauge bosons. The chiral model of electroweak and strong interactions is described by massless fields. The third pillar is the mechanism with which these fields acquire mass, the Higgs mechanism. Finally, the interactions happen and the calculations are performed using a $3+1$ dimensional space-time structure.

Apart from the so far missing Higgs boson, the SM has survived all the tests that the current precision measurements could offer. The compatibility between theory and experiment is yet unshaken. The SM can even be trivially amended to accommodate the recently established existence of the neutrino masses.

However, there are a number of reasons hinting that the SM can not be the final theory of fundamental particles and their interactions. The fine tuning problem in the Higgs mass, the non-unification of electro-weak and strong forces, the arbitrariness of the fermion masses and mixings, the origin of the number of generations, and the source of baryo-genesis are such hints.

The ATLAS experiment [1] is being setup to investigate possible solutions to the above mentioned problems of the SM . It will take data at the LHC accelerator using $7+7 \mathrm{TeV}$ proton beams. The accelerator is expected to accumulate about $1 \mathrm{fb}^{-1}$ integrated luminosity by the end of 2009 and then to gear up to a low luminosity regime at which $10 \mathrm{fb}^{-1}$ per year will be collected. The experimental setup is currently being commissioned with technical and cosmic runs [2].

${ }^{a}$ e-mail: gokhan.unel@cern.ch 


\section{Beyond the Standard Model}

The theories proposed to solve the problems of the SM change its fundamental structure. These changes will be searched for with the ATLAS detector to validate or refute the theories Beyond the SM (BSM). This note classifies the BSM theories according to the modifications they bring to the SM. It will subsequently give the reach of ATLAS for some BSM theories as a function of the energy scale, the model parameters at which the new physics emerge and the integrated luminosity collected by the experiment.

\subsection{Searches for new Matter}

A possible change to the SM is the modification of its matter content. This can be achieved either by invoking a sub-structure to the elementary particles, or by adding new elementary fermions to the known ones. The following resumes these two options.

\section{New Constituents}

It is possible to formulate a model in which known elementary particles are composed of more fundamental constituents, for example composite or preonic models. Such models predict excited fermions both charged and neutral [3]. The reach of ATLAS for excited electrons and quarks have been studied previously [1]. A more recent work (see [4] and the references therein) concentrates on the excited neutrinos, $\nu *$ which can be produced by Drell-Yan processes and decay to a boson and a lepton. The Monte Carlo study scanned the mass of $\nu *$ in the range $(0.5,2.5) \mathrm{TeV}$ and investigated the possibility to fully reconstruct the $\nu *$ invariant mass from its decay products $(\nu+Z$ or $e+W)$. The bosons were reconstructed in all decay modes except the tau decays.

The SM backgrounds originate from the decays of $p p \rightarrow W Z$ and $p p \rightarrow$ $W W W$. Fig. 1 shows the experimental reach of this signature for $300 \mathrm{fb}^{-1}$ as a function of the $\nu *$ mass and energy scale at which the compositeness appears $(\Lambda)$. The higher (lower) curve is for $3(5)$ sigma statistical significance.

\section{New Quarks}

Additional quarks are predicted by various models. The three most popular cases are down type iso-singlets, up type iso-singlets and iso-doublets. The fourth family iso-doublet quarks [5], suggested by the flavour democracy arguments, have been studied almost 10 years ago [1]. The down type iso-singlets $(D)$ are proposed by $E_{6}$ group inspired models [6]. The pair production, mostly independent of the extended CKM matrix elements, and decay of the $D$ quarks were studied recently [7] using the ATLAS fast simulation software. The mass 


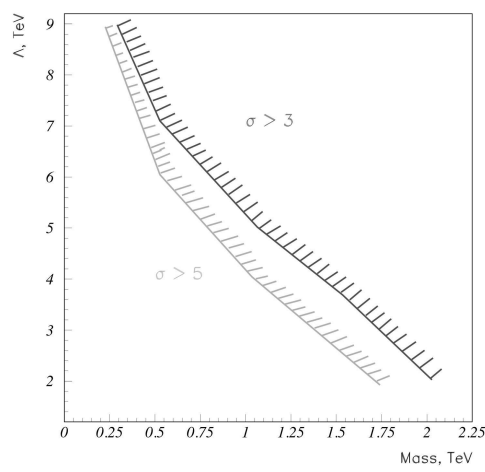

Figure 1: ATLAS reach for $\nu *$ using $300 \mathrm{fb}^{-1}$ integrated luminosity

of the new quark, which is assumed to mix with the first (or second) SM family, was scanned. The considered decay $(D \bar{D} \rightarrow Z Z d d \rightarrow 4 \ell 2 j)$ where $\ell=e, \mu$ has a low event yield but a clean signature allowing the full reconstruction of the new quark's invariant mass. The left plot of Fig. 2 shows an example reconstructed $D$ quark invariant mass peak compared to the background events originating from all SM $Z Z j j$ processes. The right plot of Fig. 2 shows the 3 sigma and 5 sigma curves as a function of the new quark mass and integrated luminosity.
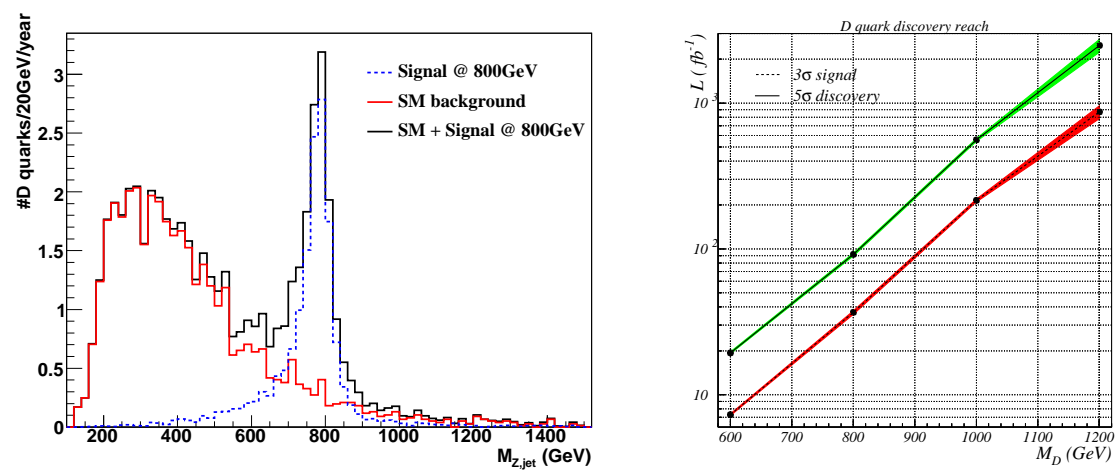

Figure 2: Search for down type iso-singlet quarks. Left: The invariant mass reconstruction for $m_{D}=800 \mathrm{GeV}$. Right: The discovery reach as a function of the quark mass; signal and discovery curves are shown.

The up type iso-singlet quarks, $T$, are predicted by the Little Higgs models [8]. The jet associated single $T$ quark production from $W$ exchange were considered 
assuming maximal mixing to the third SM family [9]. The same work studied all three possible decay modes: $T \rightarrow W b, H t, Z t$. As seen from Fig. 3 , the $Z t$ channel has small background but also a small event yield as opposed to the $W b$ channel which has an observability potential up to $2.5 \mathrm{TeV}$ in $300 \mathrm{fb}^{-1}$. The same integrated luminosity allows a 5 sigma discovery up to $1.4 \mathrm{TeV}$ reach using the $Z t$ channel.
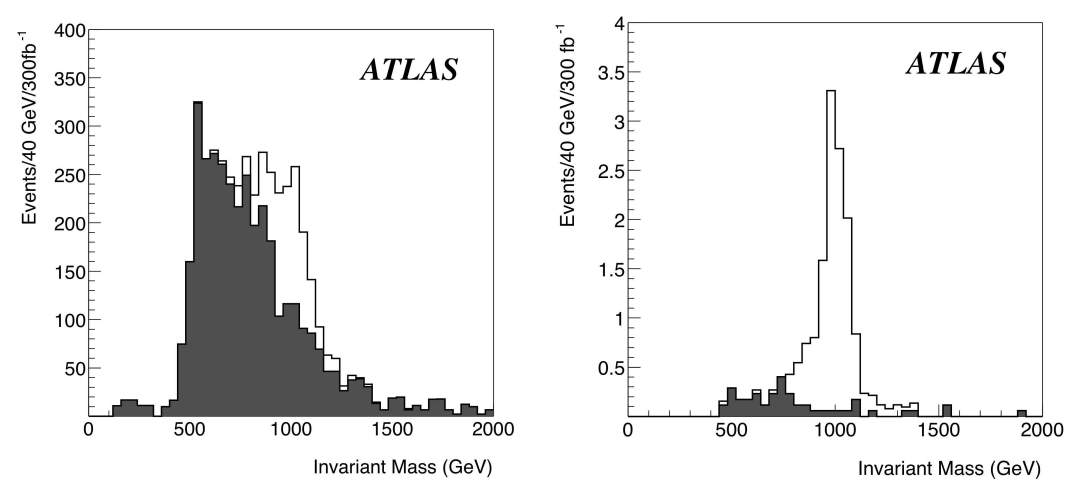

Figure 3: Invariant mass reconstruction for an up type iso-singlet quark of mass $1 \mathrm{TeV}$ using $300 \mathrm{fb}^{-1}$ integrated luminosity, $W b$ on the left and $Z t$ on the right side.

\section{New Leptons}

New leptons, $L$, appear in various models $[5,6,10]$. The work in [11] concentrates on the lepton pairs produced from quark annihilation and from gluon fusion to a quark triangular loop. In both cases, the $s$ channel contains the $Z$ boson and a possible $Z^{\prime}$ as propagator. The first one can also propagate with a $\gamma$. The considered decay mode is $L \rightarrow Z \mu / Z e$. The search was performed as a function of the new lepton and new heavy neutral gauge boson $\left(Z^{\prime}\right)$ mass. The experimental reach is given in Fig. 4 for a $Z^{\prime}$ mass of $700 \mathrm{GeV}$. The lower (upper) curve is the reach for $10(100) \mathrm{fb}^{-1}$ of integrated luminosity. A $Z^{\prime}$ of $2 \mathrm{TeV}$ would increase the $5 \sigma$ reach from $800 \mathrm{GeV}$ to $1 \mathrm{TeV}$ for $100 \mathrm{fb}^{-1}$.

\section{Leptoquarks}

Leptoquarks $(L Q)$ are predicted by GUT and composite models. The study in [12] considered their pair production from gluon fusion and quark annihilation. The same work also covers the single production. The decay modes consist of electrons (type-1) or neutrinos (type-2) and a light jet. For both scalar and vector $L Q \mathrm{~s}$, the mass scan was performed for different coupling coefficients. 


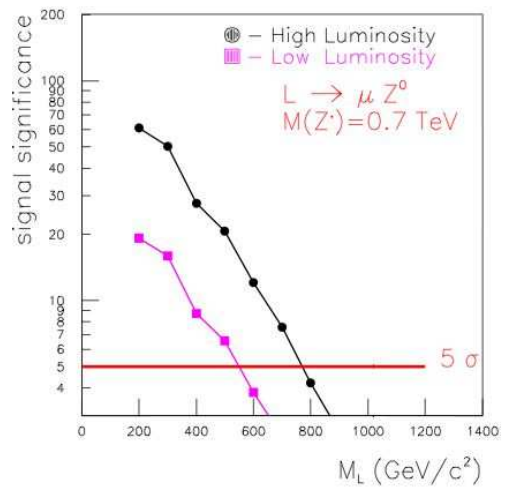

Figure 4: $L$ reach as a function of its mass, low (high) luminosity corresponds to $10(100)$ $\mathrm{fb}^{-1}$ of integrated luminosity.

Fig. 5 summarizes the reach for $300 \mathrm{fb}^{-1}$, showing that about $1.2(1.5) \mathrm{TeV}$ $L Q$ can be discovered for scalar (vector) leptoquark models.

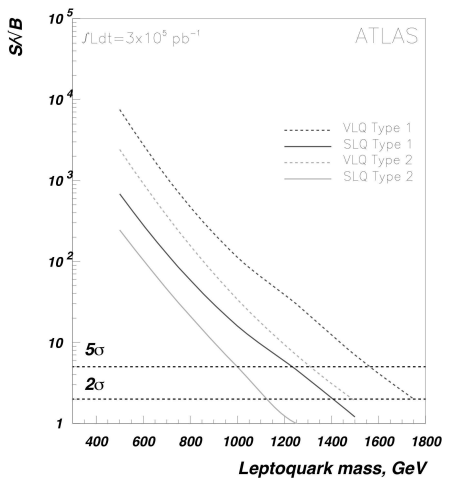

Figure 5: $L Q$ reach as a function of its mass for $300 \mathrm{fb}^{-1}$ of integrated luminosity.

\subsection{Searches for new gauge group structure}

Embedding the SM gauge group into a larger one brings additional gauge bosons, both neutral $\left(Z^{\prime}\right)$ and charged $\left(W^{\prime}\right)$. Additionally they appear in models with extra-dimensions (ED) as the Kaluza-Klein (KK) [13] excitations of their SM counterparts. 


\section{Neutral gauge Bosons}

A full GEANT MC simulation study was performed to investigate the $Z^{\prime}$ discovery potential of ATLAS using a generic parameterization called CDDT [14]. The CDDT parameterization classifies $Z^{\prime}$ searches into four distinct cases, depending on its coupling to the known fermions. In this study, a 1.5 and $4 \mathrm{TeV}$ $Z^{\prime}$ produced by quark anti-quark annihilation was allowed to decay into $e^{+} e^{-}$ pairs. The left side of Fig. 6 shows the ATLAS reach for $100 \mathrm{fb}^{-1}$ of integrated luminosity as a function of the ratio of the new gauge boson and its gauge coupling strength $\left(M_{z} / g_{z}\right)$ and fermion coupling modification parameter $(x)$. A recent study investigated the discovery reach of the KK excitations of the $Z$ boson, $Z^{n}[15]$. This model uses different parameterizations $(\mathrm{A}, \mathrm{B}, \mathrm{C})$ to reproduce the known fermion masses and mixings. The right side of Fig. 6 shows the reconstruction of the $Z^{n}$ invariant mass from $e^{+} e^{-}$pairs using a full GEANT based simulation together with the SM Drell-Yan background. The $Z^{n}$ discovery reach for $100 \mathrm{fb}^{-1}$ integrated luminosity is up to $6 \mathrm{TeV}$, depending on the parameterization.
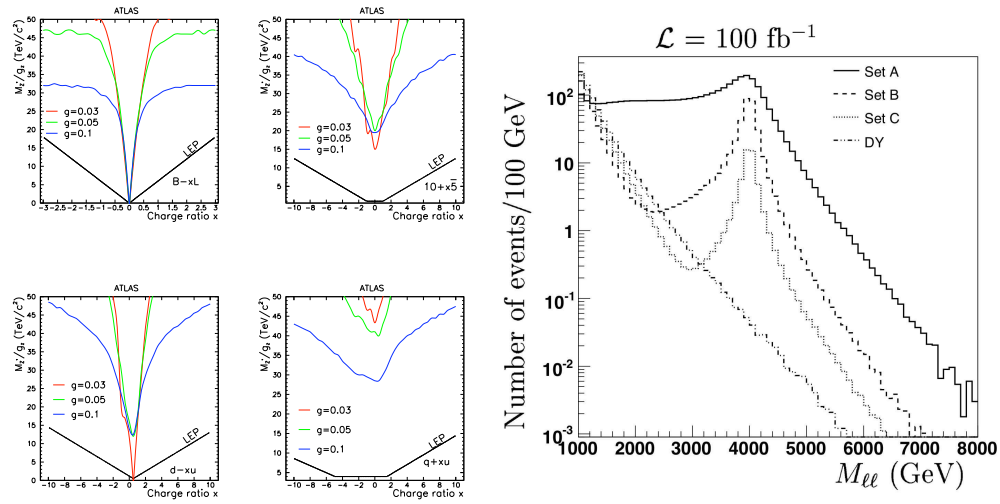

Figure 6: Left: generic $Z^{\prime}$ search with CDDT parameterization; Right: $Z^{1}$ invariant mass reconstruction for different fermion parameterizations. In both cases results with $100 \mathrm{fb}^{-1}$ are shown.

\section{Charged gauge Bosons}

Additional charged gauge bosons, $W^{\prime}$, appear in GUT, Little Higgs and ED models $[6,8,18]$. The quark anti-quark annihilation produces the $W^{\prime}$ that can be studied via its hadronic [16] or leptonic [17] decays. The important parameters are the $W-W^{\prime}$ mixing angle $(\cot \theta)$ and the mass of the $W^{\prime}$. Fig. 7 shows the discovery reach for the $W_{H}$ search (Little Higgs model), for $300 \mathrm{fb}^{-1}$ integrated luminosity in the $\cot \theta-m_{W_{H}}$ plane for the $W_{H} \rightarrow t b$ and $W_{H} \rightarrow e \nu$ modes. 


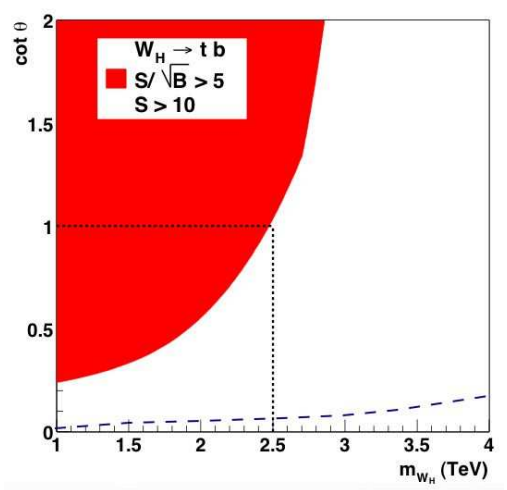

Figure 7: $W^{\prime}$ discovery reach plane. The shaded area is from hadronic decay channel, the dashed line is from electron decay.

\subsection{Searches for new Electro-weak symmetry breaking mechanisms}

Popular theories inducing fermion and boson mass generation mechanisms are Technicolor family models which were previously investigated [1], Dynamical symmetry breaking [21], variants of Higgs mechanism and supersymmetry [8, $10,19]$.

\section{New Scalars}

The spin 0 particles proposed for fermion and boson mass generation can be searched in collider experiments. The studies in the context of the Little Higgs models for chargeless and singly-charged scalars can be found in [8]. The doubly-charged scalars, $\Delta^{ \pm \pm}$predicted by both Little Higgs and left-right symmetric models (LRSM) provide a unique experimental signature with same sign leptons in the final state. A MC study considered the reach of ATLAS for $\Delta^{ \pm \pm}$singly produced from $W$ fusion and pair produced from Drell-Yan processes [20]. With all charged lepton channels separately considered, a mass scan was performed. Fig. 8 shows the reach for 100 (a) and 300 (b) $\mathrm{fb}^{-1}$ integrated luminosity. The discovery reach is about $1.8 \mathrm{TeV}$ for the single production and about $1.1 \mathrm{TeV}$ for pair production both depending on the masses of the additional vector bosons.

\section{Supersymmetry}

The postulated symmetry between the matter and force carrier particles would not only solve the hierarchy and fine tuning problems of particle physics but also provide a candidate for the Dark Matter (DM) searches of astrophysics [22]. 

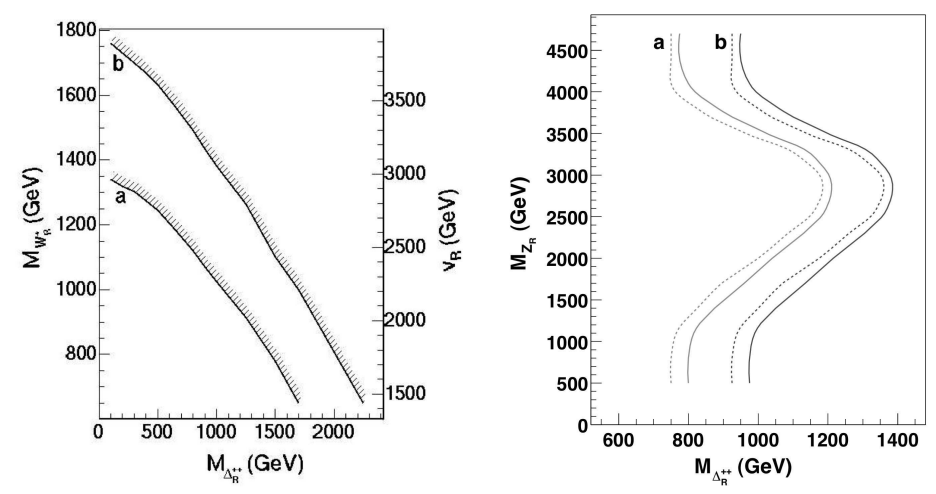

Figure 8: $\Delta^{ \pm \pm}$search reach for single production on the left and for pair production on the right.

As the SUSY particles are expected to cascade decay down to the lightest supersymmetric particle (LSP), the n jets + m leptons + missing $E_{T}$ channels are investigated. The large number of free parameters can be reduced to 5 in case of mSUGRA [23] which proposes its LSP, the lightest neutralino, $\tilde{\chi}^{0}$ as the DM candidate. However, the reduced parameter set should also be consistent with WMAP data (Fig. 9, left). A recent work has investigated the reach of ATLAS for gluino pair production in the focus point scenario and subsequent decay to $\tilde{\chi}^{0}$ and $t \bar{t}$ pairs [24]. The result of the study is shown in Fig. 9, right side, after background subtraction, for $10 \mathrm{fb}^{-1}$ of integrated luminosity providing $7 \sigma$ signal significance.
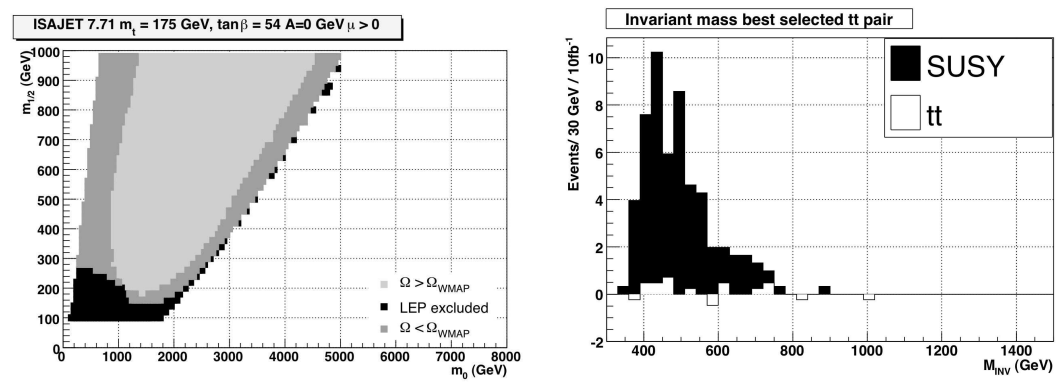

Figure 9: Left: parameter space in mSUGRA. The medium gray region is consistent with WMAP data. Right: reconstructed $\tilde{g}$ visible invariant mass for $1 \mathrm{fb}^{-1}$ of data. 


\subsection{Searches for new Dimensions}

If the relative weakness of the gravitational force is attributed to the existence of extra dimensions (ED), the graviton becomes the object to search for. The graviton couples to all particles and can escape undetected. The most promising channels are gluon-gluon, quark-gluon fusion and quark anti-quark annihilation yielding one jet + missing $E_{T}$. The experimental reach depends on the number of EDs and also the fundamental gravity scale. A study has shown that, for $100 \mathrm{fb}^{-1}$ of integrated luminosity, the reach would be about 9,7 and $6 \mathrm{TeV}$, for 2, 3 and 4 additional dimensions [25].

Large $\left(\mathrm{TeV}^{-1}\right)$ EDs appearing in ADD models [26] predict KK excitations of gluons, $g *$, which would decay into heavy quark anti-quark pairs. A study evaluated the reach of ATLAS for the decay into $b \bar{b}$ and $t \bar{t}$ pairs [27]. Depending on the mass of the $g *$, it is possible to discover a $g *$ with mass up to $3.3 \mathrm{TeV}$ with an integrated luminosity of $300 \mathrm{fb}^{-1}$.

\section{Results and Conclusions}

Although this note summarized only a selection of discovery possibilities, it has shown that ATLAS has a very rich discovery potential for physics beyond the SM. The differentiation between models and the possible boost to SM process cross sections from the particles proposed by the BSM physics were also not discussed. The preparation of the experimental apparatus for data taking is well underway, the new analyses with full simulation are also ongoing. These studies will immediately be applicable to first data from LHC.

\section{Acknowledgments}

The author would like to thank A. Studenikin for his hospitality in Moscow

and F. Ledroit and A. Parker for useful discussions. G.U.'s work is supported in part by U.S. Department of Energy Grant DE FG0291ER40679.

\section{References}

[1] ATLAS Detector and Physics Performance Technical Design Report. CERN/LHCC/99-14/15.

[2] I.Riu, ATL-SLIDE-2007-05, proceedings of 15th IEEE Real Time Conference (2007).

[3] E.J.Eichten, K.D.Lane and M.E.Peskin Phys.Rev.Lett. 50, 811 (1983); L.Abbot, E.Farhi Phys.Lett., B 101, 69 (1981).

[4] A.Belyaev, C.Leroy, R.Mehdiyev, Eur.Phys.J. C 41, 1 (2005).

[5] B.Holdom, JHEP 0608, 076 (2006); B.Holdom, JHEP 0703, 063 (2007). 
[6] F.Gursey, P.Ramond and P.Sikivie, Phys.Lett. B 60, 177 (1976); F.Gursey and M.Serdaroglu, Lett. Nuovo Cimento 21, 28 (1978).

[7] R.Mehdiyev et al., Euro.Phys.J. C. 49, 613 (2007).

[8] M.Schmaltz, Nucl.Phys. B 117, 40 (2003).

[9] G.Azuelos et al., Euro.Phys.J. C. 39, Suppl.2, 13 (2005).

[10] S.Dimopoulos Nucl.Phys. B 168, 69 (1981); E.Farhi, L.Susskind, Phys.Rev. D 20, 3404 (1979); J.Ellis et al., Nucl.Phys. B 182, 529 (1981).

[11] C.Alexa, S.Dita, ATL-PHYS-2003-014 (2003).

[12] A.Belyaev et al., J.H.E.P. 09, 005 (2005).

[13] E.Witten, it Nucl.Phys. B186, 412 (1981).

[14] F.Ledroit, B.Trocme, ATL-PHYS-PUB-2006-024, proceedings of TeV 4 LHC workshop, (2006).

[15] F.Ledroit, G.Moreau, J.Morel, J.H.E.P. 09, 071 (2007).

[16] Gonzlez de la Hoz, S; March, L; Ros, E; ATL-PHYS-PUB-2006-003.

[17] G.Azuelos et al., Eur.Phys.J. C 39, 13 (2005).

[18] L.Randall, R.Sundrum, Phys.Rev.Lett. 83, 3370 (1999).

[19] I.F.Ginzburg, M.Krawczyk, Phys.Rev. D72, 115013 (2005).

[20] G.Azuelos, K.Benslama, J.Ferland, J.Phys. G 32, 73 (2006).

[21] Y.Hosotani, Phys.Lett. B 126, 309 (1983) ; B.McInnes, J.Math.Phys. 31, 2094 (1990).

[22] H.P.Nilles, Phys. Rev. 110, 1 (1984) and references therein.

[23] A.H.Chamseddine, R.Arnowitt and P.Nath, Phys.Rev.Lett. 49 (1982) 970; H.P.Nilles, Phys.Rept. 110 (1984).

[24] U.De Sanctis, T.Lari, S.Montesano, C.Troncon, arXiv:0704.2515, SNATLAS-2007-062, Eur.Phys.J. C52, 743 (2007).

[25] L.Vacavant, I.Hinchliffe, J.Phys. G 27, 1839 (2001).

[26] N.Arkani-Hamed, S.Dimopoulos, G.Dvali, it Phys.Lett. B 429, 96 (1998).

[27] L.March, E.Ros, B.Salvachua, ATL-PHYS-PUB-2006-002 (2006). 